\title{
Light Responsiveness of the Suprachiasmatic Nucleus: Long-Term Multiunit and Single-Unit Recordings in Freely Moving Rats
}

\author{
Johanna H. Meijer, ${ }^{1}$ Kazuto Watanabe, ${ }^{2}$ Jeroen Schaap, ${ }^{1}$ Henk Albus, ${ }^{1,3}$ and László Détári ${ }^{4}$ \\ ${ }^{1}$ Department of Physiology, Leiden University Medical Centre, 2300 RC Leiden, The Netherlands, ${ }^{2}$ Department of \\ Physiology, Dokkyo University School of Medicine, 321-02 Mibu, Japan, 3/nstituut voor Epilepsiebestrijding, 2100AA \\ Heemstede, The Netherlands, and ${ }^{4}$ Department of Comparative Physiology, Eötvös Loránd University, \\ $\mathrm{H}-1088$ Budapest, Hungary
}

The suprachiasmatic nuclei (SCN) of the hypothalamus contain a pacemaker that generates circadian rhythms in many functions. Light is the most important stimulus that synchronizes the circadian pacemaker to the environmental cycle. In this paper we have characterized the baseline neuronal firing patterns of the SCN as well as their response to light in freely moving rats. Multiunit and single-unit recordings showed that SCN neurons increase discharge during daytime and decrease discharge at night. Discharge levels of individual neurons that were followed throughout the circadian cycle appeared in phase with the population and were characterized by low discharge rates (often below $1 \mathrm{~Hz}$ ), with a twofold increase during the day. The effect of light on the multiunit response was dependent on the duration of light exposure and on light intensity, with light thresholds of $\sim 0.1$ lux. The light response level showed a strong dependency on time of day, with large responsiveness at night and low responsiveness during day. At both phases of the circadian cycle, the response level could be raised by an increase in light intensity. Single-unit measurements revealed that the time-dependent light response of SCN neurons was present also at the level of single units. The results show that the basic light response characteristics that were observed at the multiunit level result from an integrated response of similarly behaving single units. Research at the single-unit level is therefore a useful approach for investigating the basic principles of photic entrainment.

Key words: suprachiasmatic nucleus; circadian; entrainment; photic; rat; multiunit activity; single cells; electrophysiology
Substantial evidence indicates that the suprachiasmatic nuclei (SCN) of the hypothalamus function as a circadian pacemaker for many behavioral and physiological functions in mammals (Moore and Eichler, 1972; Rusak and Zucker, 1979; Meijer and Rietveld, 1989; Ralph et al., 1990). In constant conditions, these functions have rhythms slightly different from $24 \mathrm{hr}$ and are "free running." To adopt the environmental periodicity, the SCN require light input at specific times of the day. At the beginning of the night, light produces phase delays of the circadian pacemaker, whereas at the end it produces phase advances (Pittendrigh and Daan, 1976). The magnitude of light-induced phase delays and advances is dependent on light intensity and on duration of light exposure (Nelson and Takahashi, 1991; Meijer et al., 1992). During the day, light does not induce shifts in the pacemaker's phase.

Several neuronal pathways transmit light information from the eyes to the SCN. Of these, the direct retinohypothalamic tract is the most important for photic entrainment (Moore, 1973; Cassone et al., 1988). Other visual pathways arise from the intergeniculate leaflet (Harrington et al., 1987), the pretectum (Mikkelsen and Vrang, 1994), and possibly the raphe nuclei

\footnotetext{
Received March 20, 1998; revised Aug. 11, 1998; accepted Aug. 14, 1998.

This work was supported by the Nederlandse Organisatie voor Wetenschappelijk Onderzoek (Visiting Grant B91-248 to K.W.) and the European Community (Grant ERB-CIPA-CT92-2277). We thank Mary Harrington and Benjamin Rusak for their useful comments on this manuscript, and we thank Jan Janse and Hans Duindam for engineering and technical assistance.

Correspondence should be addressed to Dr. J. H. Meijer, Department of Physiology, P.O. Box 9604, 2300 RC Leiden, The Netherlands.

Copyright (C) 1998 Society for Neuroscience $0270-6474 / 98 / 189078-10 \$ 05.00 / 0$
}

(Foote et al., 1978). All pathways send their information to the ventral and sometimes also to the lateral borders of the SCN.

Single-unit recordings in anesthetized hamsters and rats have indicated that $\sim 30 \%$ of SCN neurons are light responsive, with a majority that is light-activated and a minority that is lightsuppressed (Groos and Mason, 1980; Meijer et al., 1986). These neurons are mainly located within the retinorecipient areas of the SCN. They give sustained responses and code for light intensity according to a sigmoid-shaped curve. However, thresholds for light responses in these neurons were found to be higher by two magnitudes than the threshold for behavioral phase shifting (Meijer et al., 1986). This discrepancy has been attributed to the use of anesthetics, which presumably reduce the sensitivity of SCN neurons to light (Dyer and Rigdon, 1987; Meijer et al., 1992). A further, major problem with anesthetics is their putative effect on the pacemaker's functioning (Colwell et al., 1993). Consequently, light response of SCN neurons cannot be studied as a function of circadian time (CT) in the presence of anesthetics.

In a previous paper, we reported our first data on the light responses of SCN neurons in freely moving (i.e., unanesthetized) rats and found maximum responsiveness during the night (Meijer et al., 1996). In the present paper we characterize SCN light response as a function of circadian phase, light intensity, and light duration. The results demonstrate that at any phase, the combination of light intensity and circadian phase determine the final response level.

Long-term single-unit recordings have so far been performed only in cultured systems (Mirmiran et al., 1995; Welsh et al., 1995; Herzog et al., 1997). In these studies it was found that individual SCN neurons have the capacity to oscillate. The importance of in 
vivo recordings was recently indicated in a study that showed differences in the characteristics of electrical multiunit activity (MUA) discharge rhythms in vivo versus in vitro (Meijer et al., 1997). Because of stabilization difficulties, it has never been possible to perform long-term recordings of single neurons in freely moving animals. We have now performed nine long-term single-unit recordings in freely moving animals for $>48 \mathrm{hr}$, and we demonstrate for these neurons a low-amplitude rhythm in spontaneous discharge rate with increased discharge during the day. Moreover we show that individual SCN neurons exhibit oscillations in their light response with maximum responsiveness during the night.

\section{MATERIALS AND METHODS}

Male Wistar rats were implanted with tripolar electrodes (Formvar insulated stainless steel, diameter $0.125 \mathrm{~mm}$ ) (Plastics One, Roanoke, VA). Two electrodes were aimed at the $\mathrm{SCN}$, with an interelectrode distance of $0.4 \mathrm{~mm}$. The third electrode was cut shorter and its insulation was partly removed before it was placed in the white matter for reference. After the surgery, the animals were put back in the colony room with a light regimen of $12 \mathrm{hr}$ light (100-150 lux) alternating with $12 \mathrm{hr}$ dark for at least $10 \mathrm{~d}$.

At the onset of the experiment, the animals were transferred to the experimental room and connected to the recording system. A flexible cable attached to a counterbalanced swivel system was used to minimalize any effect on the animal's freedom of movement. Recordings were performed through one electrode at a time. The details of recording have been described previously (Meijer at al., 1996). Briefly, after appropriate amplification and filtering (500 Hz-5 kHz), multiunit activity was converted to pulses by window discriminators and was counted by a computer every $10 \mathrm{sec}$. Signal-to-noise ratio varied over different recordings (Fig. 1). The first window discriminator was set at such a level that signal-to-noise ratio varied from 2:1 to 8:1 or more. The second window discriminator was used to isolate a single unit from the multiunit signal to record the unit for at least two circadian cycles. This was only possible when one of the cells that made up the multiunit signal was very close to the electrode tip and therefore produced spikes of large and distinctive amplitude (Fig. 1). A second criterion was uniformity of the shape of the spike during the recording session.

A third window discriminator was set at a higher level to record artifacts that occurred as a result of vigorous head movements and feeding behavior. The amplitudes of these artifacts were much larger than the amplitude of the action potentials. Those $10 \mathrm{sec}$ bins in which artifacts occurred were excluded from the analysis.

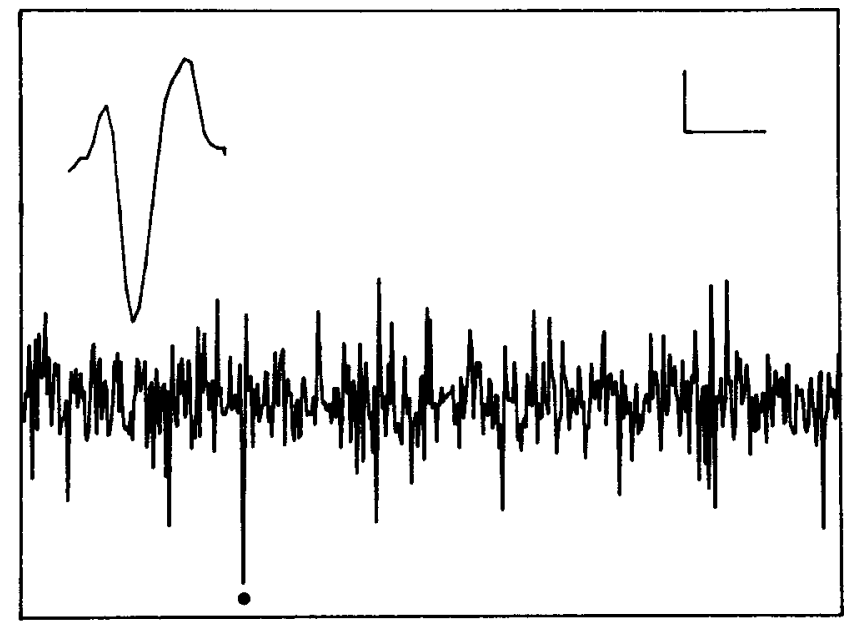

Figure 1. Representative example of an oscilloscope trace with multiunit activity. The spike indicated by a dot could be separated from the other spikes and could be recorded for several days. The inset shows the characteristic waveform of this spike. Horizontal calibration: $5 \mathrm{msec}$ (oscilloscope trace) and $0.3 \mathrm{msec}$ (inset). Vertical calibration: $10 \mu \mathrm{V}$ throughout.
Recordings were performed in constant darkness. To characterize the response of the SCN, light pulses of various intensity and duration were applied. The timing of the light pulse applications was controlled by a computer, whereas light intensities were regulated by a series of neutral density filters. To determine SCN light response as a function of CT, 6 min light pulses were applied every hour. Because this protocol is expected to affect circadian period, the animal's drinking rhythm was recorded throughout the experiment. The $\mathrm{CT}$ of light responses was calculated per day, on the basis of the onset of the drinking activity (CT 12).

At the end of the experiment, animals were deeply anesthetized with Nembutal and a positive current $(20 \mu \mathrm{A}$ for $50 \mathrm{sec})$ was passed through one of the electrodes to deposit a small amount of iron at the recording site. After this procedure, rats were perfused through the heart with physiological saline followed by a $4 \%$ Formalin solution. The Formalin solution contained $1.5 \%$ potassium ferrocyanide to obtain a blue spot by Prussian blue reaction with the iron deposit. The brain was removed and blocked to prepare freeze-cut sections of $40 \mu \mathrm{m}$. Sections were stained with cresyl violet for final inspection.

The presence of rhythmicity in the MUA activity was investigated by Lomb periodogram analysis for nonequidistant data (i.e., data sets with missing values). The level of statistical significance was assessed by a Monte Carlo simulation (Lomb, 1976; Horne and Baliunas, 1986). To quantify light responses, the steady-state response level was taken. This implies that transient increases in activity at the onset of light pulses and transient decreases in firing rate that were correlated with the animal's behavior were excluded from the analysis.

\section{RESULTS}

In the present study, 149 successful hypothalamic recordings were performed for at least one circadian cycle in 104 rats. In 52 cases, no circadian variation was seen in the multiunit activity. In the remaining 97 cases, a significant circadian rhythm was present $(p<0.01)$. Of these, 27 recordings showed a rhythm with high discharge rate during the day and low discharge rate during the night (Fig. 2). This discharge pattern is opposite to the animal's behavioral activity cycle. Histology confirmed in these rats that the recording site was within the SCN, with the exception of a few cases in which the blue spot could not be found in the sections. Reversed rhythms with high activity during the night and low activity during the day were recorded in 70 cases. Recording sites were located lateral, anterior, or posterior from the $\mathrm{SCN}$, with a maximum distance of $0.8 \mathrm{~mm}$ to the SCN border. In 17 recordings we succeeded in recording a single unit for more than $48 \mathrm{hr}$, which met the criterion for a single unit. Nine units were located in the SCN, and eight were located outside the SCN.

\section{SCN light responsiveness}

After a light stimulus, two multiunit response types were recorded inside the SCN. In most cases $(n=21)$ light induced an increase in discharge; in four cases no light response occurred, and in two cases a decrease was observed (Fig. 3). The lightactivated response usually started with a fast transient increase in MUA activity. The discharge rate then gradually decreased, and after a few minutes a steady-state response was seen. Similarly, the termination of the light pulse very often induced a sudden drop in the activity, which decreased well below the baseline level. The original activity level returned within a few minutes (see Figs. $5 A-C, 6)$. These three components of the light-activated response usually occurred together, but they also could appear separately. The pattern of the light-depressed response was very different from the light-activated ones. No transients were seen, but instead there was a gradual decrease in MUA activity when the light was turned on. The discharge rate reached the steadystate level after 1-2 min. A similar, slow buildup of activity was observed at the end of the light pulse.

The effect of stimulus duration on the response pattern was 
A

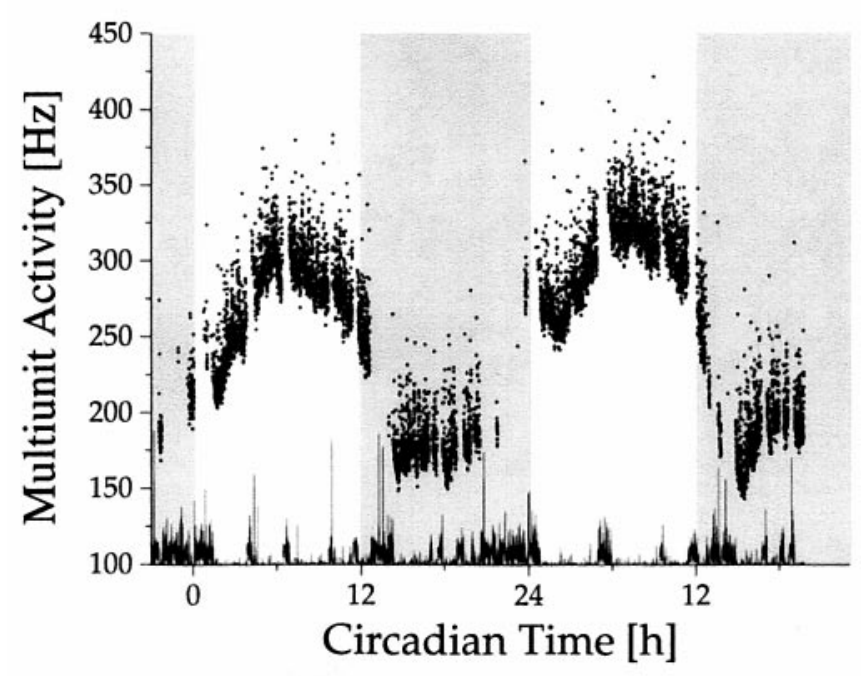

B

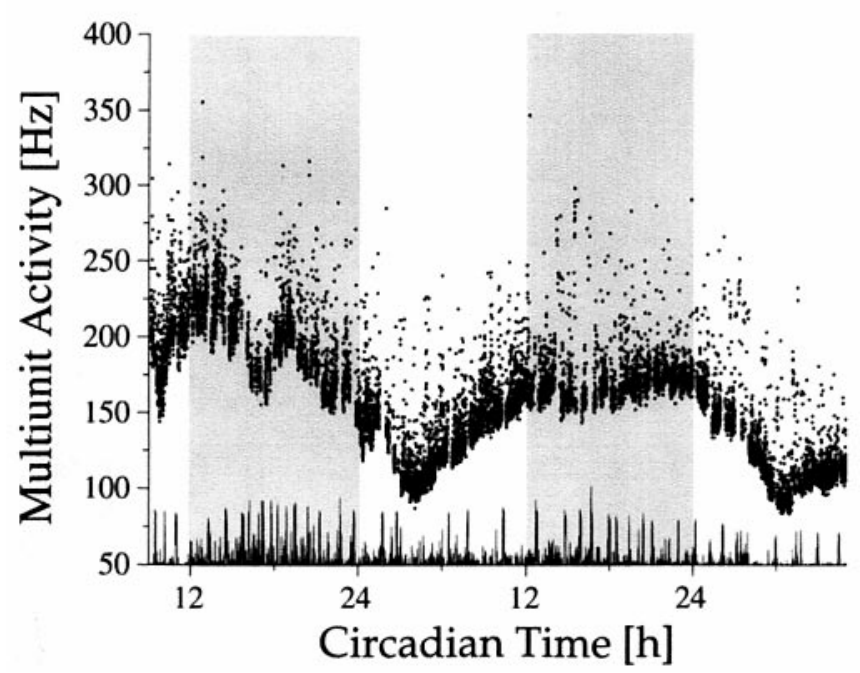

Figure 2. Long-term multiunit activity as a function of circadian time. Circadian time was determined on the basis of drinking actograms. The recordings were performed in constant darkness. The onset of drinking activity is the onset of the subjective night and corresponds with CT 12. The subjective night is shaded. Multiunit discharge inside $(A)$ and outside $(B)$ the SCN was counted every $10 \mathrm{sec}$. Dots represent mean values per minute. At the bottom of each figure, movement artifacts have been plotted. The gaps in the multiunit traces are the consequence of the exclusion of data bins in which movement artifacts occurred.

investigated by presenting light pulses of different length ranging from 2 to $30 \mathrm{~min}$ (Fig. 4). Responses appeared sustained in all cases and lasted for the full duration of the light stimulus. The amplitude of the response was usually constant, but in a few instances a small, gradual increase or decrease was seen.

To investigate the effect of light intensity on discharge level, 6 min light pulses were used at different light intensities ranging from $10^{-4}$ to 140 lux. The light intensities were spread evenly on a logarithmic scale. At any circadian time a monotonic relation was found between light intensity and neuronal discharge. The monotonic relation holds for both the light-activated and the light-suppressed responses (Fig. 5). To quantify light response of SCN neurons as a function of light intensity, circadian time had to be taken into account.
A

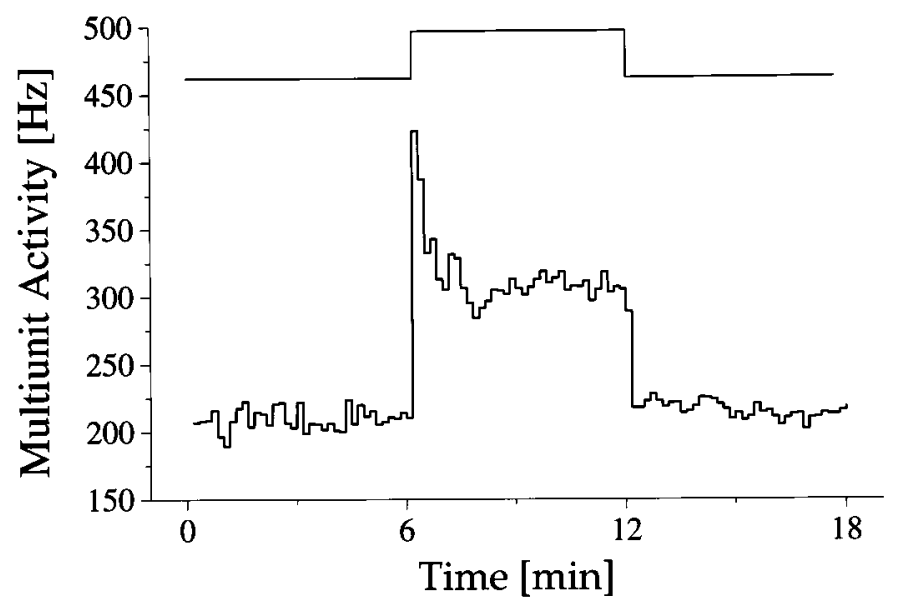

B

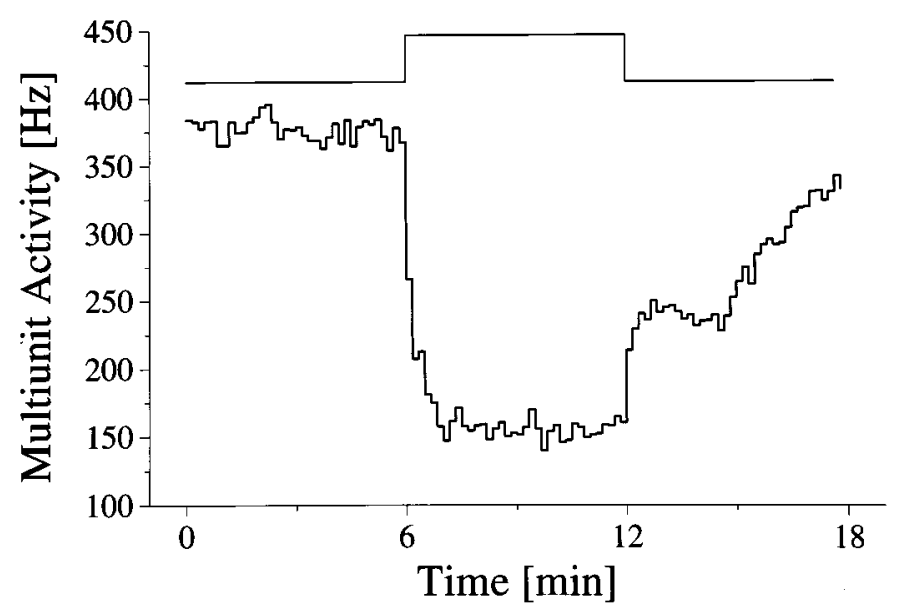

Figure 3. Multiunit light response of SCN neurons. The timing of the light pulse is indicated in the step diagram above the records. The light-activated $(A)$ and light-suppressed $(B)$ responses represent the mean of five and seven responses, respectively, obtained during two circadian cycles.

\section{Rhythmic light response}

When light response was studied as a function of circadian time, a significant difference in light response emerged between day and night (Fig. 6). During the day, the baseline discharge was relatively high, with a maximum at mid subjective day. Light presentations during this phase elicited only small responses. During the night, when baseline discharge was in its lower phase, large light responses were obtained. The variation in light response emerged not as a sudden transition in responsiveness but as a gradual process (Fig. 7A), and it displayed significant circadian rhythmicity $(p<0.001)$. From mid subjective day onward, a steady increase in responsiveness was observed lasting until late subjective night. From there a fast decline started, leading to small responsiveness during mid subjective day. The steady-state multiunit response to a light pulse applied at night exceeded the light response during the day (in all but one cases), despite the fact that baseline activity during night was lower. The direction of the response never changed in the course of the day.

The threshold of the light responses was $\sim 0.1$ lux at any phase 


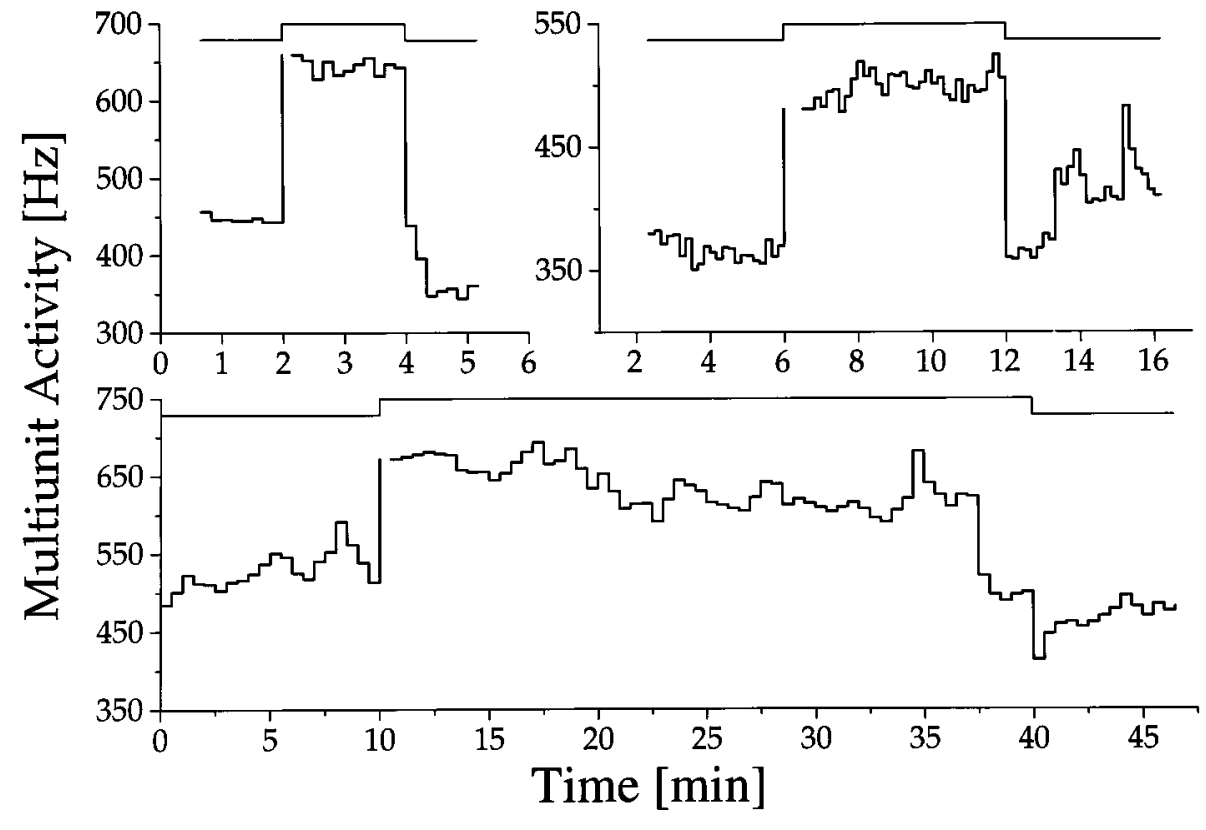

Figure 4. Responses to light pulses of different duration. Light responses of SCN neurons are sustained for 2, 6, and 30 min light pulses. The drop in discharge at the end of the 30 min light pulse is accidental. Discontinuity in the data, immediately after the onset of a light pulse, resulted from movement artifacts.

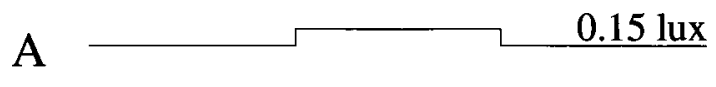

$265 \mathrm{~Hz}$

B

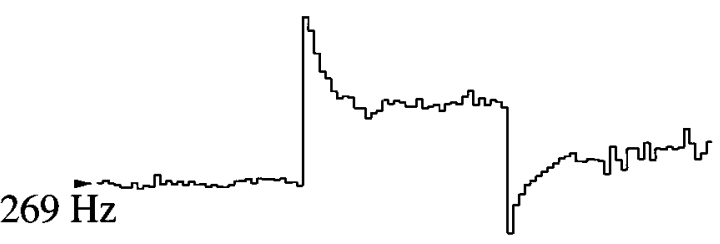

2 lux

140 lux

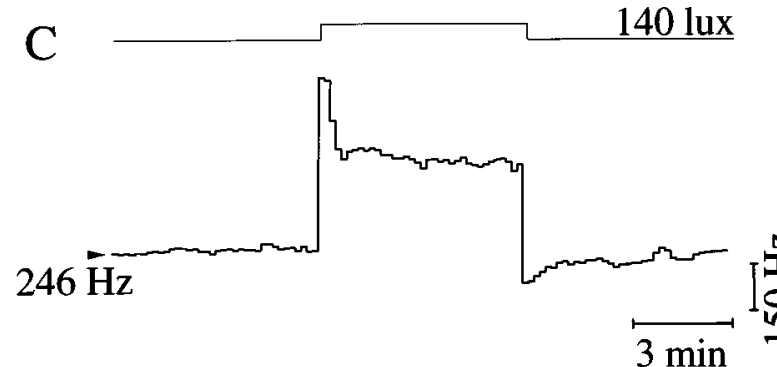

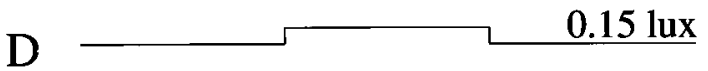

$243 \mathrm{~Hz}$

$\mathrm{E}$

$2 \operatorname{lux}$
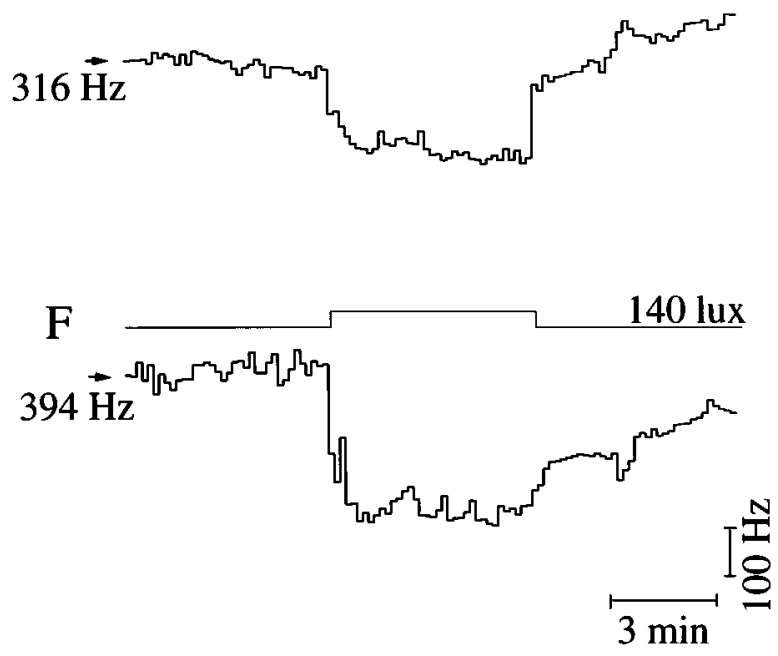

Figure 5. Light responses to increasing light intensities. Activated $(A-C)$ and suppressed $(D-F)$ response types with three to five traces averaged per light intensity. Intensities are indicated above the step diagram.

of the circadian cycle. When light intensity was increased, the change in response depended on the circadian time. At mid subjective night, when sensitivity was high, responses grew rapidly as a function of light intensity, and saturation occurred between 1 and 10 lux. At mid subjective day, responses increased only slowly as a function of intensity, and saturation occurred above 100 lux (Fig. 8).

A complete description of the steady-state light response should be based on its dependence on light intensity and CT at any phase of the circadian cycle. We have exposed the animals to 


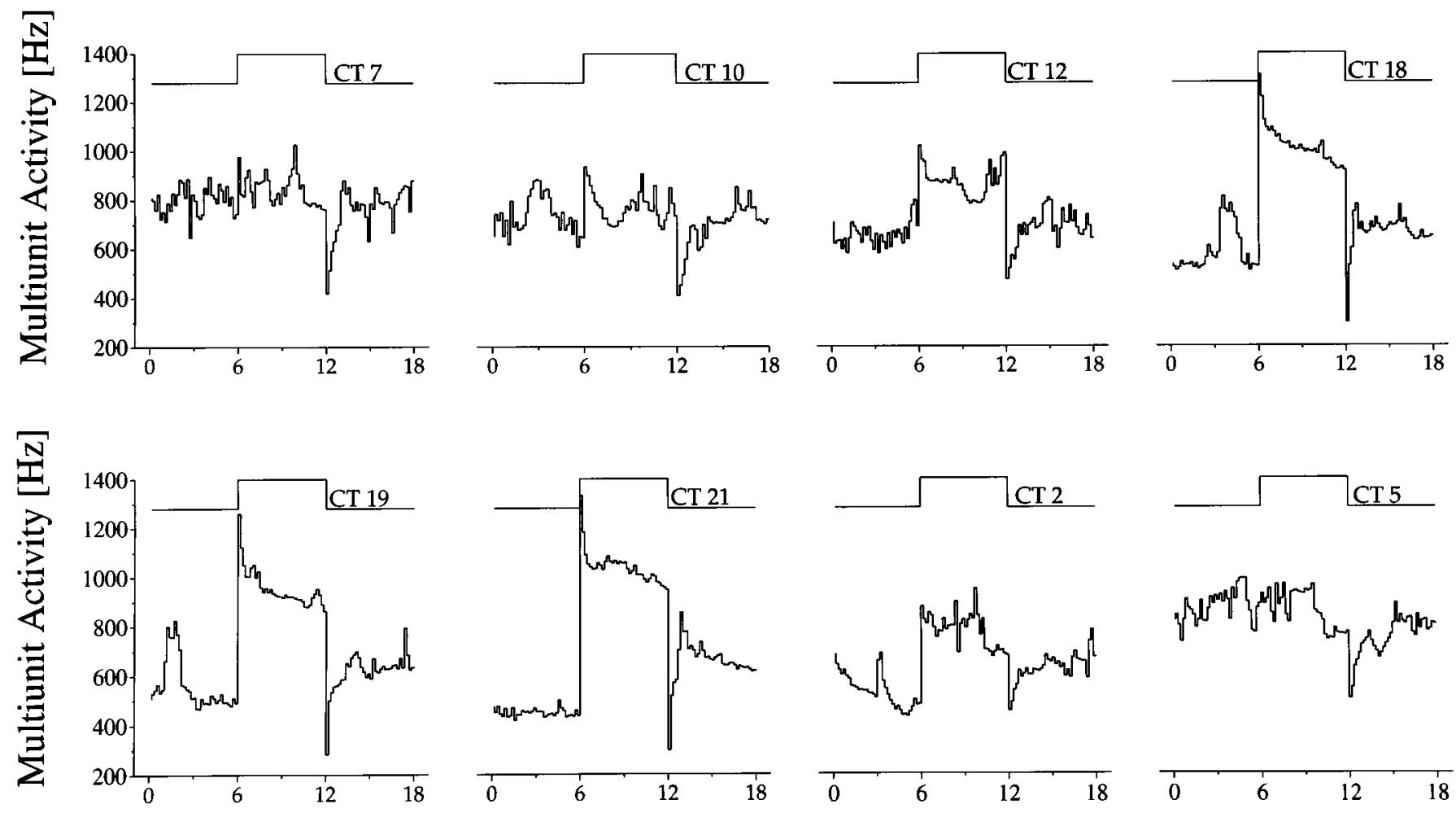

Figure 6. Light responses as a function of circadian time. Light intensity was set at 0.15 lux. Above the step diagram, circadian time $(C T)$ of each response has been given. The change in magnitude of the light response throughout the circadian cycle is clearly visible, as is the circadian rhythm in basal MUA.

different light intensities on consecutive circadian cycles. Often, one light intensity was presented for two circadian cycles. The results indicate that at any circadian phase the response of the SCN is determined by the combination of light intensity and circadian time (Fig. 9).

Outside SCN-type rhythms were obtained in 70 recordings, with 23 cases showing light responsiveness. These light responses were relatively weak and always of the light-activated type. When light response was measured as a function of circadian time, high responses appeared during mid subjective night and low responses appeared during mid subjective day (Fig. 7B). Thus light sensitivity rhythms were in the same phase inside and outside the $\mathrm{SCN}$, whereas baseline activity rhythms were in opposite phase (Fig. 10).

\section{Single-unit recordings}

Long-term single-unit recordings in which an individual unit could be recorded for at least $48 \mathrm{hr}$ were performed in 17 cases, nine inside the SCN and eight outside the SCN (Fig. 11). In all SCN neurons, baseline discharge patterns of single units showed increased activity during the day and decreased activity during the night, with no exception (Table 1 ). The mean discharge rate of SCN neurons at mid subjective night was $0.7 \mathrm{~Hz}$ and ranged from 0.01 to $4 \mathrm{~Hz}$, depending on the neuron. In seven of nine neurons, discharge did not exceed $0.2 \mathrm{~Hz}$ during the night. The mean discharge during mid subjective day was $1.39 \mathrm{~Hz}$ and ranged from 0.02 to $8 \mathrm{~Hz}$. In seven of nine cases, discharge did not raise above $0.7 \mathrm{~Hz}$ during the day.

In four long-term recordings of single SCN units, lightactivated responses were observed. All light-activated SCN neurons showed a significant circadian rhythm in light response, with high responsiveness during night and low responsiveness during day $(p<0.001)$. In fact, the rhythm in light response was more pronounced than the rhythm in baseline discharge (Fig. 12). SCN light response during the day reached a mean value of $7.15 \mathrm{~Hz}$ and during the night a mean value of $13.05 \mathrm{~Hz}$.

Outside the SCN none of the eight neurons exhibited significant circadian discharge patterns in baseline firing frequencies, and none of the neurons were light responsive. The mean discharge rate outside the SCN was $0.44 \mathrm{~Hz}$.

\section{DISCUSSION}

\section{Baseline rhythms}

This is the first study in which the behavior of single SCN neurons was followed throughout the circadian cycle and could be linked to the animal's circadian activity rhythm. Single-unit analysis was performed only in those cases in which a neuron that was apparently close to the recording electrode generated a spike that was much larger than all others in the multiunit signal. If the shape of this spike varied, however, we supposed that it was generated by more than one neuron, and consequently single-unit analysis was not possible. On the basis of these criteria, we demonstrated the presence of circadian rhythmicity in the discharge pattern of nine individual SCN neurons. For all of these neurons, discharge was high during day and low at night, which is similar to the multiunit discharge pattern. Previous long-term recordings in cultured systems (Mirmiran et al., 1995; Herzog et al., 1997) and in dissociated cultured systems (Welsh et al., 1995) have indicated rhythms in spontaneous discharge, but no relation with subjective day or night could be made. In two of these studies, neurons were recorded simultaneously and found to differ occasionally in phase 

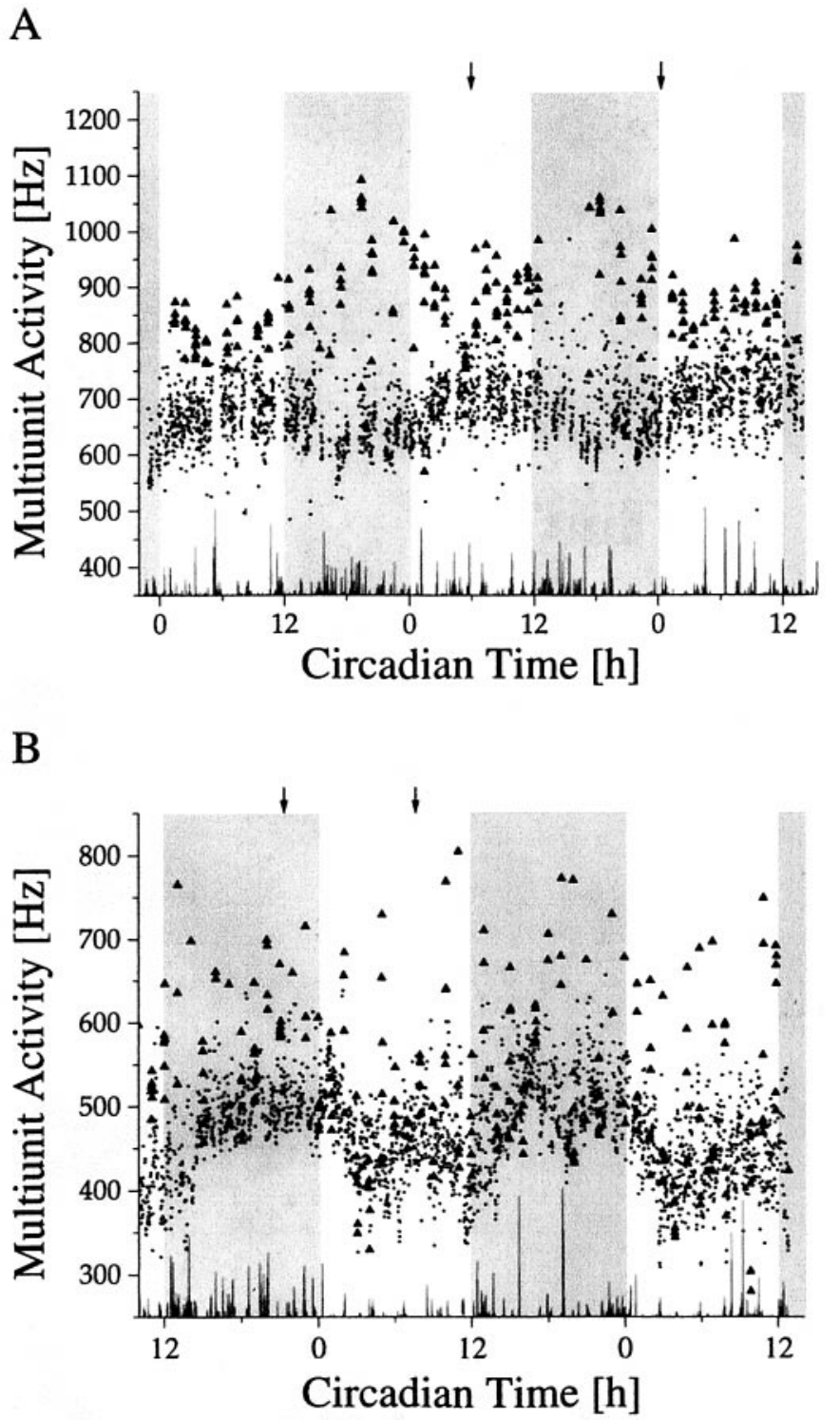

Figure 7. Light responses during long-term multiunit activity recordings inside $(A)$ and outside $(B)$ the SCN. The subjective night of the animal is shaded. Light pulses were given every hour for $6 \mathrm{~min}$, with an intensity of 0.15 lux. In between the light presentations, the animal was in total darkness. The mean discharge rates per minute during the periods of darkness are indicated by a dot. The mean discharge rates per minute during the light intervals are indicated by triangles. Light responses marked by an arrow are shown on a shorter time scale in Figure 10.

or period, especially in the dissociated cultured system. In contrast, our single units were always synchronized with the multiunit discharge pattern, which suggests that they were also mutually synchronized. It is possible that dissociated cultured systems lack essential possibilities to synchronize (Welsh et al., 1995). Nevertheless we feel that the number of single-unit recordings in vivo is too small to generalize at this point and additional measurements are required.

As an important difference with isolated in vitro preparations, we found that discharge rates were very low, in most cases below $1 \mathrm{~Hz}$. In vitro studies generally describe discharge rates between 1 and $10 \mathrm{~Hz}$ (Green and Gillette, 1982; Groos and Hendriks, 1982; Shibata, 1982; Thomson et al., 1984; Mason, 1991). Possibly the absence of inhibitory afferents in vitro (such as those from the

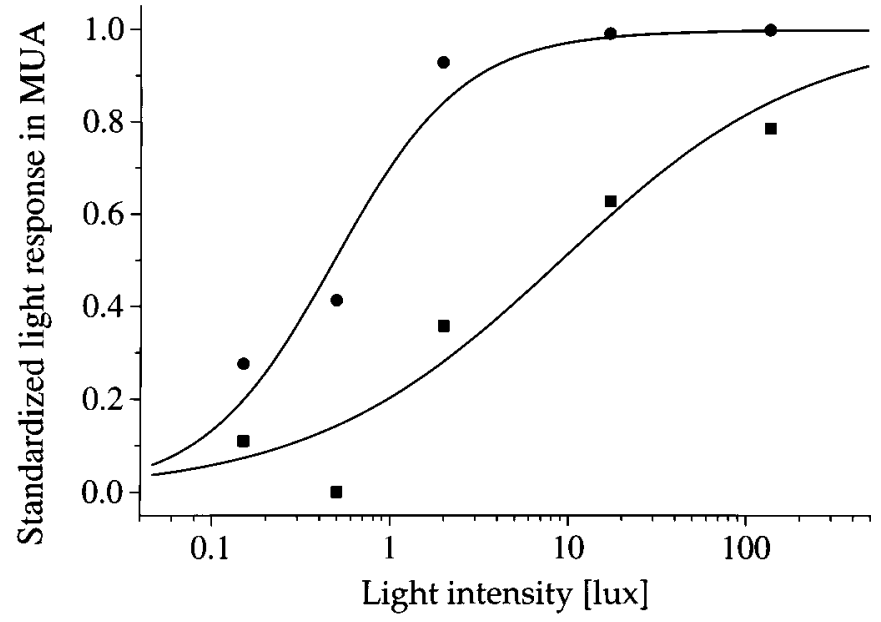

Figure 8. Averaged light sensitivity of activated SCN neurons during day $(\boldsymbol{\square})$ and night $(\bullet)$. The maximum light response obtained in each animal was normalized to 1 . The standardized light response is plotted against the light intensity and was obtained by taking the difference between baseline activity in the dark immediately before the light pulse, and discharge during light presentation. The difference was averaged for the mid subjective day $\pm 3 \mathrm{hr}(\boldsymbol{\square})$ and for mid subjective night $\pm 3 \mathrm{hr}(\mathbf{)})$. Data points were fitted with a Michaelis equation: $y=x^{\mathrm{a}} / x^{\mathrm{a}}+b^{\mathrm{a}}$. The fitted parameters were $a=0.6, b=9$ lux and $a=1.2, b=0.5$ lux for the day and the night, respectively.

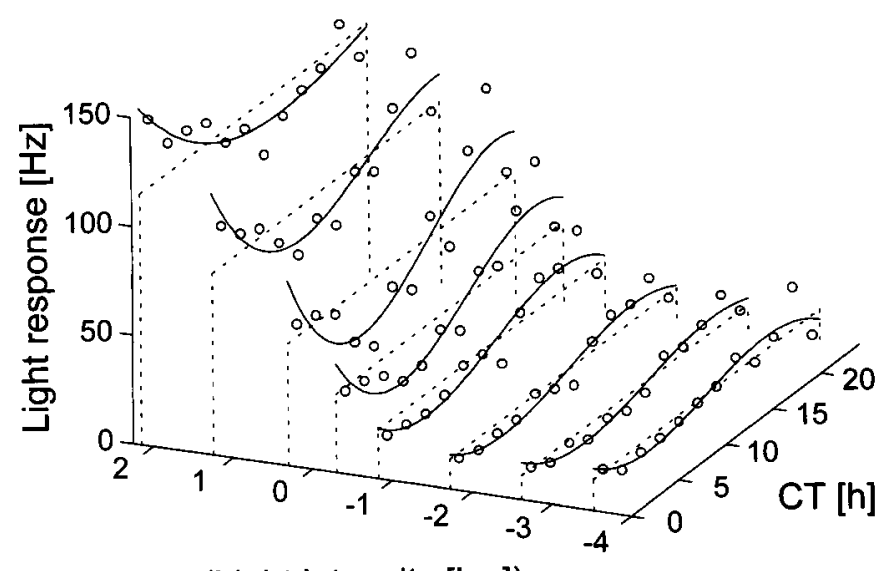

$\log$ (Light intensity [lux])

Figure 9. Light response of SCN neurons as a function of both light intensity and circadian time in a particular animal. The averaged response level was plotted against circadian time (assessed by the drinking rhythm) and against the logarithm of light intensity (in lux). The response level was determined by taking the difference between the dark discharge level before the light pulse and the discharge level during light and was averaged per $2 \mathrm{hr}$. For every light intensity used, the circadian variation in light response has been fitted by a free three-order polynomial, whereas the average is indicated by a dashed line, to enable visualization.

raphe and geniculate nucleus) lead to increases in firing rate. Other factors such as increased concentrations of potassium and calcium in the artificial medium may further increase neuronal discharge.

A second difference with dissociated cultured systems is the shape of the circadian discharge pattern. In all of our neurons, discharge during daytime increased approximately twofold. This amplitude is less than those obtained with multiunit recordings in vitro (Bouskila and Dudek, 1993; Meijer et al., 1997). In vitro, the system is deafferented from the brain, which results in a loss of 

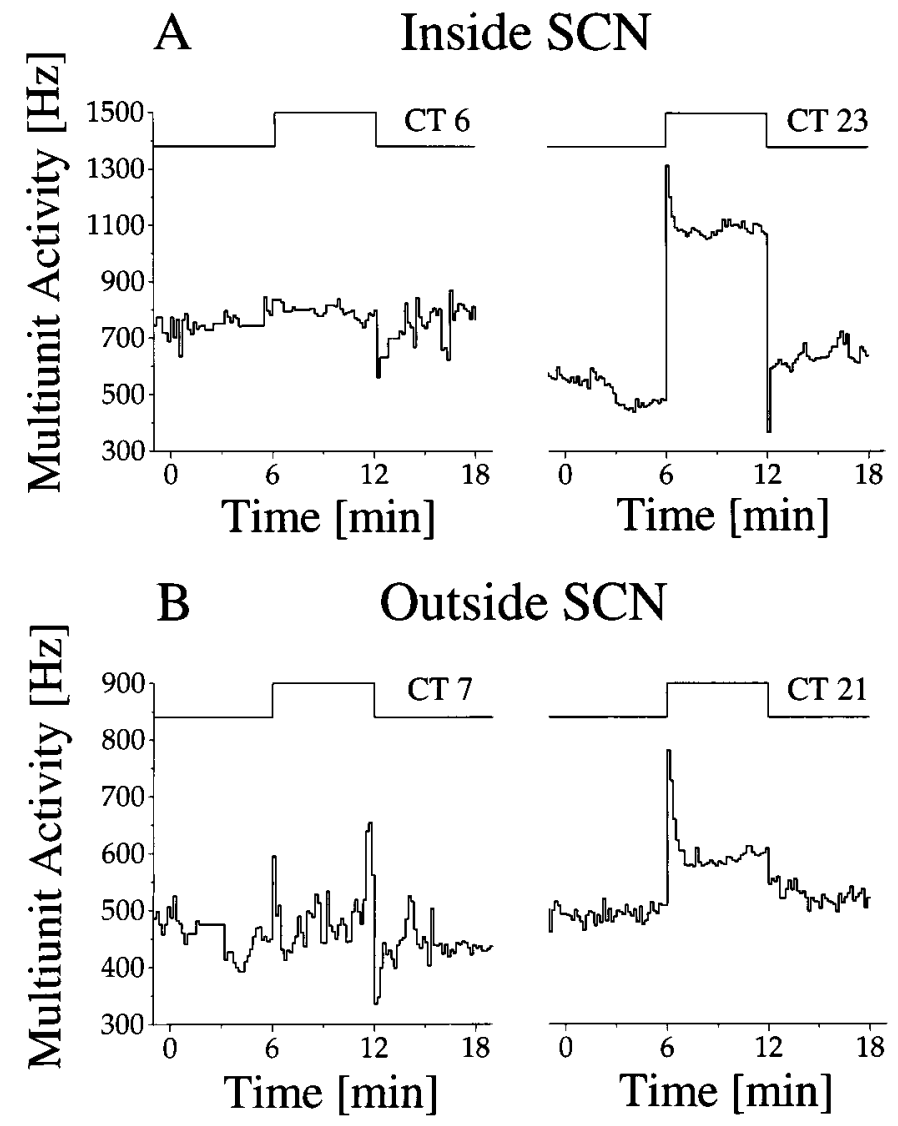

Figure 10. Light response inside $(A)$ and outside $(B)$ the SCN. Circadian time of the light pulses is specified above the step diagram. The long-term recording traces from which these examples are taken are given in Figure $7 A, B$. The timing of the light pulses is indicated by the arrows in Figure 7. Circadian rhythms in baseline discharge inside and outside the SCN are in opposite phase. Nevertheless, the rhythm in light response is similar inside and outside the SCN. Furthermore, it can be observed that light responses in the SCN were larger than outside the SCN, which was a general observation.

synaptic input. Local interactions may be somewhat reduced as well. In dissociated culture systems, cellular interactions are expected to be even lower. In such cultures, the rhythm in discharge appeared even more pronounced and increased 5- to 10-fold in the course of the cycle (Welsh et al, 1995). In the cell culture system of Herzog et al. (1997), amplitudes varied among the various examples. In those cases in which synchronized cell firing rhythms were shown in the figures, rhythm amplitude was smaller as compared with those instances in which cells did not fire in synchrony. Our results suggest that a decrease in cellular communication leads to a decrease in synchronization and to an increase in amplitude of an individual neuron's firing rhythm.

\section{SCN light response}

Almost all multiunit recordings from inside the SCN (23 of 27) showed clear responses to light. These responses were positive, with the exception of two recordings in which a decrease in discharge was observed. Short-term recordings have indicated that most light-responsive neurons are activated by light and only a minority are light-suppressed. Therefore, it is expected that light-activated responses dominate the response pattern in multiunit recordings in which hundreds of neurons are recorded simultaneously. The presence of a light-suppressed response in two of our multiunit recordings may indicate a clustering of cell types within the SCN.

Retinal input to the SCN has been shown to use glutamate as a transmitter (DeVries at al, 1993, 1994; Ding et al., 1994). Excitatory amino acids are known to have fast actions on the firing behavior of neurons, which is in good agreement with the abruptness of the light-activated responses observed in the present experiments. On the other hand, the slow buildup and decay of the light-suppressed responses suggest that different pathways are responsible for this response type.

The direction of response never changed in the course of the circadian cycle. In other words, both the light-suppressed and the light-activated responses retained their sign. These results extend an important study of Inouye (1984) in which light-activated responses were similar at four different times of the circadian cycle. This phenomenon, which can be examined only in longterm recordings, is of critical importance in understanding photic entrainment.

Apparently no relation exists between the positive or negative sign of the photic response and the advance/delay zone of the pacemaker. Even during subjective day, when light stimuli do not phase shift the pacemaker, light responses could be obtained in $\mathrm{SCN}$ neurons. For yet unknown reasons, neuronal discharge does not impinge on the pacemaker during daytime.

In this respect, the organization of the circadian pacemaker appears similar to the organization of the pacemaker in the marine snail Bulla gouldiana (Block et al., 1993). Electrophysiological recordings in this snail have shown that throughout the circadian cycle, pacemaker cells respond to light with an increase in discharge, despite the fact that phase delays and advances are obtained only during the first and second part of the subjective night, respectively, as is the case in mammals. However, depolarization results in an opening of calcium channels only during the night when these channels are normally closed. It remains to be determined whether calcium plays a similar role in the phaseresetting mechanism of the mammalian pacemaker. Current lines of investigation will reveal whether calcium plays a similar role in the more complex circadian system of mammals (van den Pol et al., 1992; Chen and van den Pol, 1998).

We have demonstrated that light-activated responses in the multiunit signal could also be measured outside the SCN, although these were much weaker than inside the SCN. This is consistent with the observation that hypothalamic areas adjacent to the SCN also receive retinal innervation, but this is relatively sparse (Levine et al., 1991). Light-suppressed responses were never observed outside the SCN.

\section{Rhythmic light response}

Although light responses are present throughout the circadian cycle, they are not constant. During daytime, when baseline activity is high, the light response of the SCN is low. In the night, when baseline activity is low, light response is high. Thus, the increase in light response occurs when baseline decreases. Noticeably, the light response pattern outside the SCN was also circadian, with large responsiveness at night. Thus outside the $\mathrm{SCN}$, the rhythm in light response follows the rhythm in baseline discharge, and high responsiveness coincides with the phase when baseline activity is high.

In a previous paper we speculated that the increased light responsiveness at night might be the result of the hyperpolarized membrane potentials of SCN neurons at this phase of the cycle, resulting in increased driving force and therefore increased light 


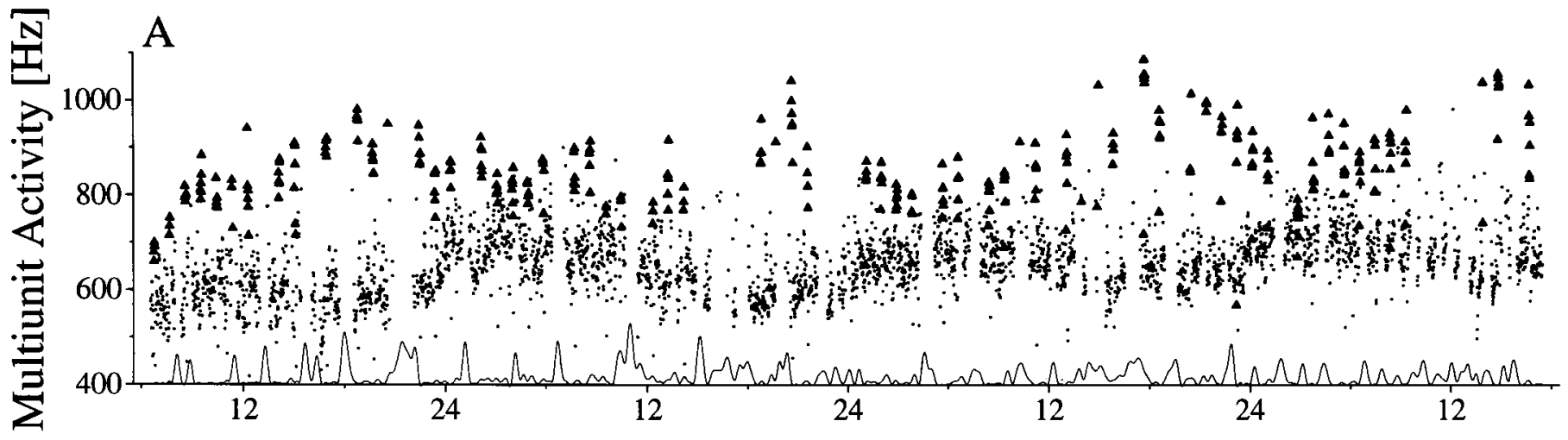

\section{Circadian Time [h]}
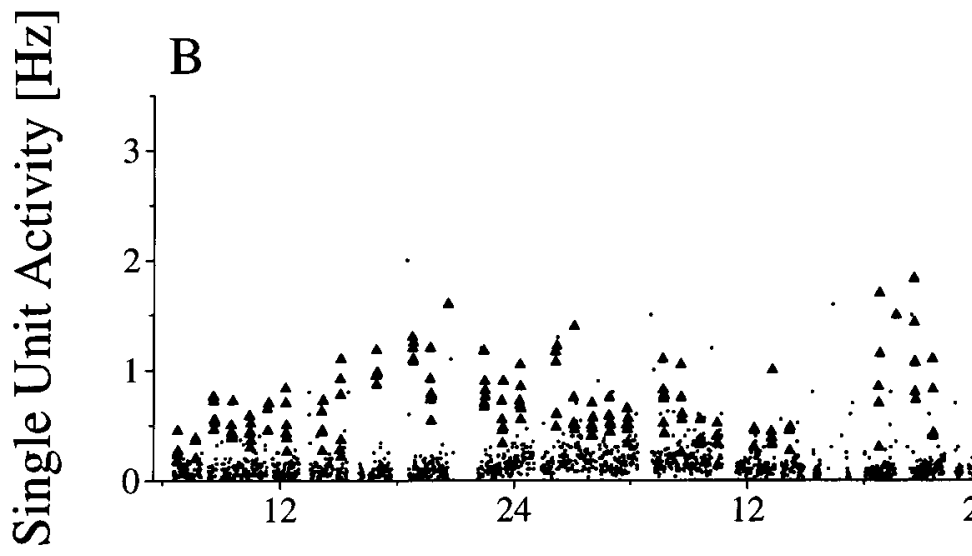

12

24

12

24

\section{Circadian Time $[\mathrm{h}]$}

Figure 11. Simultaneous multiunit and single unit recording. A, Multiunit baseline discharge pattern (๑) and discharge rate during hourly 6 min light presentation $(\mathbf{\Lambda})$. The trace at the bottom indicates the occurrence of movement artifacts. $B$, Single-unit baseline discharge $(\boldsymbol{\bullet})$ varies between a mean of $0.05 \mathrm{~Hz}$ during night and $0.2 \mathrm{~Hz}$ during day. The light response level $(\boldsymbol{\Lambda})$ varies between mean levels of $\sim 1 \mathrm{~Hz}$ during night and $0.5 \mathrm{~Hz}$ during day. After a recording time of $\sim 36 \mathrm{hr}$ the single unit was lost. Circadian time 12 corresponds to the onset of drinking activity.

Table 1. Discharge rates of individual SCN neurons during the subjective day and night when the animal was in darkness

\begin{tabular}{llllcc} 
& \multicolumn{2}{l}{ Baseline $(\mathrm{Hz})$} & & \multicolumn{2}{c}{ Light response $(\mathrm{Hz})$} \\
\cline { 2 - 3 } Animal number & Day & Night & & Day & Night \\
\hline 80b013 & 0.05 & 0.02 & & 0.1 & 0.2 \\
90b001 & 2.5 & 1.5 & 8 & 16 \\
66a003 & 0.4 & 0.2 & 20 & 35 \\
45a016 & 0.2 & 0.05 & 0.5 & 1 \\
73b010 & 0.02 & 0.01 & & \\
74b001 & 0.25 & 0.15 & & \\
U99b001 & 0.7 & 0.15 & & \\
U99a001 & 8 & 4 & & \\
U97a001 & 0.35 & 0.2 & & \\
Mean & 1.39 & 0.70 & 7.15 & \\
Mean of ratio & \multicolumn{2}{l}{$2.47: 1$} &
\end{tabular}

The ratio of discharges was calculated per neuron, before the mean was established. Of the nine single units, four appeared responsive to light pulses of 0.15 lux. Presented are the steady-state light response levels obtained during 6 min light presentations during the subjective day and night.

response (Meijer et al., 1996). The present recordings from areas surrounding the SCN suggest instead that the rhythm in light response is independent from the baseline discharge rhythm.
Therefore, the origin of the light-response rhythm should be upstream from the $\mathrm{SCN}$, along the light-input pathway.

Light-responsive SCN cells receive information from retinal ganglion cells. In the ocular pacemaker of the rat, circadian rhythms have been observed in the expression of transducin, which mediates the initial stages in the phototransduction cascade (Brann and Cohen, 1987). Furthermore, disk shedding and electroretinogram sensitivity exhibit circadian rhythms (LaVail, 1980; Besharse, 1982; White and Hock, 1990; for review, see Remé et al., 1991). Also in the isolated retina, circadian rhythms have been observed in melatonin production (Tosini and Menaker, 1996). So far, it is not known whether these ocular rhythms have consequences for the processing of light information. The present findings are a first indication that retinal pacemakers modulate light transmission to the brain and have functional importance.

Conclusive evidence was obtained for rhythmicity in light response of single SCN neurons. Light responses of single units showed increased responsiveness at night and low responsiveness during day. Thus, the light-response pattern of individual SCN neurons was similar to the pattern observed in multiple unit recordings. The results indicate that the electrophysiological characteristics of the SCN are present also at the level of the individual neurons. Therefore, long-term singleunit recording is a promising new approach for learning more 


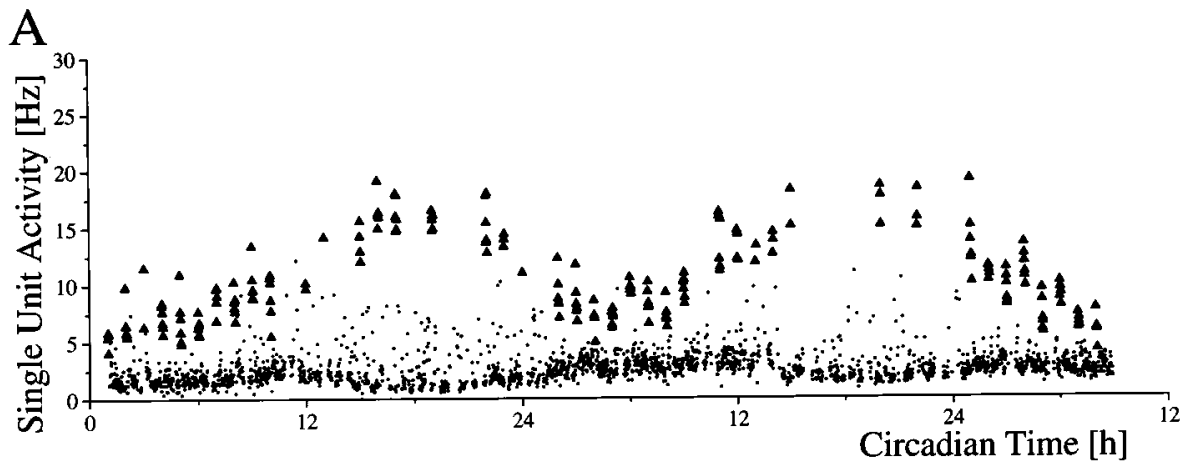

Figure 12. Long-term single-unit recording inside the SCN. A, Two circadian cycles of multiunit baseline activity indicated per minute in dots, with mean values of $\sim 1.5 \mathrm{~Hz}$ during night and $2.5 \mathrm{~Hz}$ during day. Responses to hourly light pulses of $6 \mathrm{~min}, 0.15$ lux are indicated per minute by triangles and vary between $16 \mathrm{~Hz}$ during night and $8 \mathrm{~Hz}$ during day. Circadian time 12 corresponds to the onset of drinking activity of the animal. $B$, Example of a light response during daytime at a different scale. The example is taken from the recording depicted in $A$ and represents the mean response during mid subjective day (CT $6 \pm 2 \mathrm{hr}$ ). $C$, Light response during mid subjective night (CT $18 \pm 2 \mathrm{hr})$.
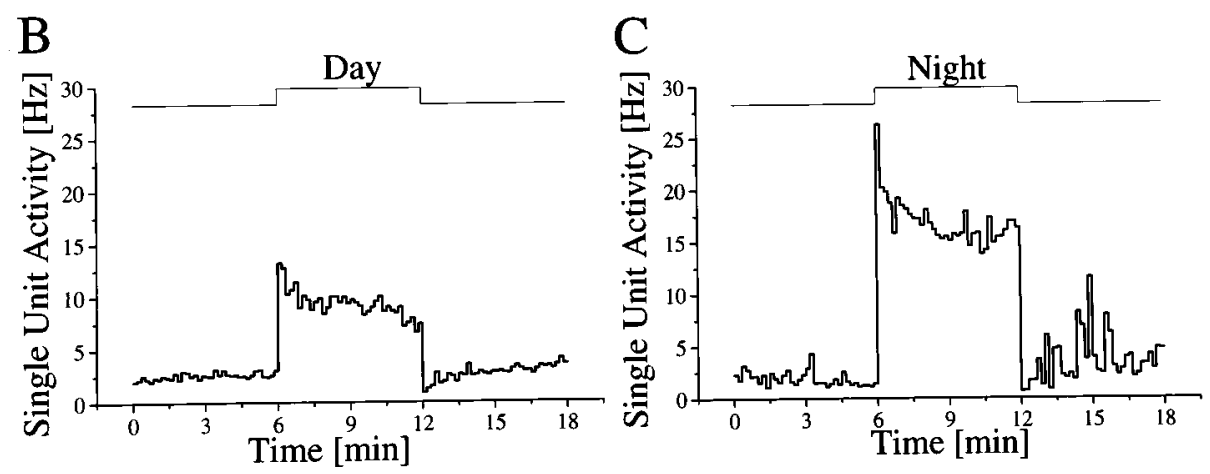

about the organization and synchronization of the circadian pacemaker.

\section{REFERENCES}

Besharse JC (1982) The daily light-dark cycle and rhythmic metabolism in the photoreceptor-pigment epithelial complex. In: Progress in retinal research, Vol 1 (Osborne N, Chader G, eds), pp 81-124. Oxford: Pergamon.

Block GD, Khalsa SBS, McMahon DG, Michel S, Guesz M (1993) Biological clocks in the retina: cellular mechanisms of biological timekeeping. Int Rev Cytol 146:83-144.

Bouskila Y, Dudek FE (1993) Neuronal synchronisation without calcium dependent synaptic transmission in the hypothalamus. Proc Natl Acad Sci USA 90:3207-3210.

Brann MR, Cohen LV (1987) Diurnal expression of transducin mRNA and translocation of transducin in rods of rat retina. Science 235:585-587.

Cassone VM, Speh JC, Card JP, Moore RY (1988) Comparative anatomy of the mammalian hypothalamic suprachiasmatic nucleus. J Biol Rhythms 3:71-91.

Chen C, van den Pol AN (1998) Presynaptic GABA B $_{\text {autoreceptor mod- }}$ ulation of P/Q-type calcium channels and GABA release in rat suprachiasmatic nucleus neurons. J Neurosci 18:1913-1922.

Colwell CS, Kaufman CM, Menaker M, Ralph MR (1993) Lightinduced phase shifts and Fos expression in the hamster circadian system: the effects of anaesthetics. J Biol Rhythms 8:179-188.

De Vries MJ, Nunes Cardozo B, Vander Want J, De Wolf A, Meijer JH (1993) Glutamate immunoreactivity in terminals of the retinohypothalamic tract of the brown Norwegian rat. Brain Res 612:231-237.

De Vries MJ, Treep JA, Pauw de ESD, Meijer JH (1994) The effects of electrical stimulation of the optic nerves and anterior optic chiasm on the circadian activity rhythm of the Syrian hamster: involvement of excitatory amino acids. Brain Res 642:206-212.

Ding JM, Chen D, Weber ET, Faiman LE, Rea MA, Gillette MU (1994) Resetting the biological clock: mediation of nocturnal circadian shifts by glutamate and NO. Science 266:1713-1717.

Dyer RS, Rigdon GC (1987) Urethane affects the rat visual system at subanesthetic doses. Physiol Behav 41:327-330.

Foote WE, Taber-Pierce E, Edwards L (1978) Evidence for a retina projection to the midbrain of the cat. Brain Res 156:135-140.

Green DJ, Gillette R (1982) Circadian rhythm of firing rate recorded from single cells in the rat suprachiasmatic brain slice. Brain Res 245:198-200.

Groos GA, Mason R (1980) The visual properties of rat and cat suprachiasmatic neurons. J Comp Physiol 135:349-356.

Groos GA, Hendriks J (1982) Circadian rhythms in electrical discharge of rat suprachiasmatic neurons recorded in vitro. Neurosci Lett 34:283-288.

Harrington ME, Nance DM, Rusak B (1987) Double-labelling of neuropeptide Y-immunoreactive neurons which project from the geniculate to the suprachiasmatic nuclei. Brain Res 410:275-282.

Herzog ED, Geusz ME, Khalsa SBS, Straume M, Block GD (1997) Circadian rhythms in mouse suprachiasmatic nucleus explants on multimicroelectrode plates. Brain Res 757:285-290.

Horne JH, Baliunas SL (1986) A prescription for period analysis of unevenly sampled time series. Astrophys J 302:757-763.

Inouye ST (1984) Light responsiveness of the suprachiasmatic nucleus within the island with the retino-hypothalamic tract spared. Brain Res 294:263-268.

LaVail MM (1980) Circadian nature of rod outer segment disc shedding in the rat. Invest Ophthalmol 19:407-411.

Levine JD, Weiss ML, Rosenwasser AM, Miselis RR (1991) Retinohypothalamic tract in the female albino rat: a study using horseradish peroxydase conjugated to cholera toxin. J Comp Neurol 306:344-360.

Lomb NR (1976) Least-squares frequency analysis of unequally spaced data. Astrophys Space Sci 39:447-462.

Mason R (1991) The effects of continuous light exposure on Syrian hamster suprachiasmatic (SCN) neuronal discharge activity in vitro. Neurosci Lett 123:160-163.

Meijer JH, Rietveld WJ (1989) Neurophysiology of the suprachiasmatic circadian pacemaker in rodents. Physiol Rev 69:671-707.

Meijer JH, Groos GA, Rusak B (1986) Luminance coding in a circadian pacemaker: the suprachiasmatic nucleus of the rat and the hamster. Brain Res 382:109-118.

Meijer JH, Rusak B, Gänshirt G (1992) The relation between lightinduced discharge in the suprachiasmatic nucleus and phase shifts of hamster circadian rhythms. Brain Res 598:257-263.

Meijer JH, Watanabe K, Détàri L, Schaap J (1996) Circadian rhythm in light response in suprachiasmatic nucleus neurons of freely moving rats. Brain Res 741:352-355.

Meijer JH, Schaap J, Watanabe K, Albus H (1997) Multiunit activity 
recordings in the suprachiasmatic nuclei: in vivo versus in vitro models. Brain Res 753:322-327.

Mikkelsen JD, Vrang N (1994) A direct pretectosuprachiasmatic projection in the rat. Neuroscience 62:497-505.

Mirmiran M, Koster-van Hoffen GC, Bos NPA (1995) Circadian rhythm generation in the cultured suprachiasmatic nucleus. Brain Res Bull 38:275-283.

Moore RY (1973) Retinohypothalamic projection in mammals: a comparative study. Brain Res 49:403-409.

Moore RY, Eichler VB (1972) Loss of circadian adrenal corticosterone rhythm following suprachiasmatic nucleus lesions in the rat. Brain Res 42:201-206.

Nelson DE, Takahashi JS (1991) Sensitivity and integration in a visual pathway for circadian entrainment in the hamster (Mesocricetus auratus). J Physiol (Lond) 439:115-145.

Pittendrigh CS, Daan S (1976) A functional analysis of circadian pacemakers in nocturnal rodents. IV. Entrainment: pacemaker as clock. J Comp Physiol 106:291-331.

Ralph MR, Foster RG, Davis FC, Menaker M (1990) Transplanted suprachiasmatic nucleus determines circadian period. Science 247: 975-978.

Remé CE, Wirz-Justice A, Terman M (1991) The visual input stage of the mammalian circadian pacemaking system: I. Is there a clock in the mammalian eye? J Biol Rhythms 6:5-29.

Rusak B, Zucker I (1979) Neural regulation of circadian rhythms. Physiol Rev 59:449-526.

Shibata S, Oomura Y, Kita H, Hattori K (1982) Circadian rhythmic changes of neuronal activity in the suprachiasmatic nucleus of the rat hypothalamic slice. Brain Res 247:154-158.

Thomson AM, West DC, Vlachonikolis IG (1984) Regular firing patterns of suprachiasmatic neurons maintained in vitro. Neurosci Lett $52: 329-334$

Tosini G, Menaker M (1996) Circadian rhythms in cultured mammalian retina. Science 272:419-421.

van den Pol, Finkbeiner SM, Cornell-Bell A (1992) Calcium excitability and oscillations in the suprachiasmatic nucleus neurons and glia in vitro. J Neurosci 12:2648-2664.

Welsh DK, Logothetis DE, Meister M, Reppert SM (1995) Individual neurons dissociated from rat suprachiasmatic nucleus express independently phased circadian firing rhythms. Neuron 14:697-706.

White MP, Hock PA (1990) Effects of continuous darkness on electroretinographic correlates of photoreceptor disk shedding in rabbit. Soc Res Biol Rhythms Abstr 2:99. 